\title{
Evaluation of Control Strategies for Managing Supply Chain Risks using Bayesian Belief Networks
}

\author{
(presented at the 6th IESM Conference, October 2015, Seville, Spain) $\bigodot^{\circ} I^{4} e^{2} 2015$
}

\author{
Abroon Qazi ${ }^{1}$, John Quigley ${ }^{1}$, Alex Dickson ${ }^{1}$, Barbara Gaudenzi ${ }^{2}$ and Şule Önsel Ekici ${ }^{3}$ \\ ${ }^{1}$ Strathclyde Business School, University of Strathclyde, G4 0QU Glasgow, UK \\ (abroon.qazi, j.quigley, alex.dickson)@strath.ac.uk \\ ${ }^{2}$ Faculty of Economics, University of Verona, Verona, Italy \\ barbara.gaudenzi@univr.it \\ ${ }^{3}$ Industrial Engineering Department, Dogus University, Istanbul, Turkey \\ sonsel@dogus.edu.tr
}

\begin{abstract}
Supply chains have become complex and vulnerable and therefore, researchers are developing effective techniques in order to capture the complex structure of the supply network and interdependency between supply chain risks. Researchers have recently started using Bayesian Belief Networks for modelling supply chain risks. However, these models are still focused on limited domains of supply chain risk management like supplier selection, supplier performance evaluation and ranking. We have developed a comprehensive risk management process using Bayesian networks that captures all three stages of risk management including risk identification, risk assessment and risk evaluation. Our proposed new risk measures and evaluation scheme of different combinations of control strategies are considered as an important contribution to the literature. We have modelled supply network as a Bayesian Belief Network incorporating the supply network configuration, probabilistic interdependency between risks, resulting losses, risk mitigation control strategies and associated costs. An illustrative example is presented and three different models are solved corresponding to different risk attitudes of the decision maker. Based on our results, it is not always viable to implement control strategy at the most important risk factor because of the consideration of mitigation cost, relative loss and probabilistic interdependency between connected risk factors.
\end{abstract}

Keywords-supply chain risks; Bayesian Belief Networks; control strategies; risk measures

\section{INTRODUCTION}

Supply chains have become complex because of the globalization and outsourcing in manufacturing industries. Supply chain risk management (SCRM) is an active area of research that deals with the overall management of the risk events ranging across the entire spectrum of the supply chain including external risk factors. Supply chain risk is characterized by both the probability of an event and its severity given that an event occurs [1]. Following three components are found in all conceptualizations of risk [2]:

- Probability (likelihood) of the occurrence of an event that leads to realization of the risk

- Potential losses in case of realization of risk

- Significance of the consequences of losses
Bayesian Belief Networks (BBNs) have started gaining the interest of researchers in modelling supply chain risks [3]. BBNs offer a unique feature of modelling risks combining both the statistical data and subjective judgment in case of nonavailability of data [4-6]. Researchers have used BBNs to model specific domains of supply chain risks and validated these models through case studies. BBN is a graphical representation of causal relationships between variables and associated uncertainty in the dependency in terms of conditional probabilities [4]. The variables are represented by nodes while an arc (directed between two nodes) represents direct causal relationship.

\section{Research Gap and Contribution}

Existing research in SCRM has not addressed a very important issue of evaluating different combinations of mitigation strategies in a setting of interconnected network of supply chain actors, corresponding risks, resulting losses, and costs and benefits associated with potential strategies. We utilize BBNs in bridging this major research gap and evaluate different combinations of mitigation strategies. Furthermore, we also introduce new risk measures for evaluating risk exposure of the supply network and incorporate risk attitude of the decision maker in our model using the feature of utility function.

\section{Outline}

Literature review including discussion on limitations of the existing BBN based models is briefly presented in Section II. We propose new risk measures and modelling approach in Section III. An illustrative example of a supply network including the modelling parameters is presented in Section IV. Results are presented and discussed in Section V followed by the conclusion and future research presented in Section VI.

\section{LITERATURE REVIEW}

\section{Supply Chain Risk Management}

"SCRM is the management of supply chain risks through coordination or collaboration amongst the supply chain partners so as to ensure profitability and continuity" [7]. "SCRM aims to identify the potential sources of supply chain risk and implement appropriate actions to avoid or contain 
supply chain vulnerability" [8]. Vulnerability is defined as an exposure to serious disturbances from risks within the SC as well as risks external to the SC [9]. Supply chain risk is an event that may cause disruption to the flow of activities within the supply chain. Following are important themes for future research in the field of SCRM [10]:

- Holistic methods for capturing interdependencies between risk factors across entire supply network

- Organizational level studies for gauging maturity level

- Disruption propagation and reliability of an entire network

- Synergy of supply chain risk management and project risk management in new product development

- Mechanism design for mitigating strategic risks

- Supply chain risk management practices in small and medium enterprises

\section{Bayesian Belief Network based Modelling Approaches}

Few researchers have applied BBNs in SCRM. BBNs are helpful in benchmarking supplier risk profiles and identifying risk events having largest potential impact on an organization's revenues [11]. In one of the studies involving BBNs, suppliers are benchmarked against their risks based on the sensitivity analysis [12]. However, a major limitation of the study is difficulty to get data from current and potential suppliers in populating the model.

BBNs have been applied in managing risks associated with large engineering project and developing a model for quantifying the risk of project delay in Korean shipbuilding industries [13]. Combination of BBNs and Total Cost of Ownership method was used for selection of potential suppliers that demonstrated suitability of BBNs for modeling risks in case of buyer's incomplete knowledge about the suppliers [14].

\section{Limitations of existing BBN based Models}

The main limitation of all these studies is application of BBNs in specific domain of Supply Network. None of the mentioned studies has captured holistic dynamics of the interacting risks. Furthermore, the models do not segregate risks into triggers, risk events and consequences. These models do not consider two important aspects of associating loss value to each risk and considering the influence of mitigation strategies on these risks.

In a recent study [15], supply chain process and risks corresponding to various segments of the supply network are combined and modelled as a BBN. New risk measures are also proposed in order to identify important elements within the supply network. However, the computation of proposed risk measures may not be possible in case of a supply network having complex structure. Furthermore, the proposed model describes risk analysis under a given configuration whereas it is equally important to consider the impact of various risk mitigation strategies across various segments of the network.

\section{PRoposed BBN BASEd MOdEL OF A SUPPLy NETWORK}

\section{A. Mathematical Representation}

A discrete supply chain risk network $N=(X, G, P, L)$ is a four-tuple consisting of

- a directed acyclic graph $(D A G), G=(V, E)$, with nodes, $V$, representing discrete risk events, $X_{R}$, discrete control strategies, $X_{S}$, and loss functions, $L$, and directed links, $E$, encoding dependence relations

- a set of conditional probability distributions, $P$, containing a distribution, $P\left(X_{R_{i}} \mid X_{p a\left(R_{i}\right)}\right)$, for each risk event, $X_{R_{i}}$

- a set of loss functions, $L$, containing one loss function, $l\left(X_{p a(V)}\right)$, for each node $v$ in the subset $V_{l} \in V$ of loss nodes.

$$
E L(X)=\prod_{X_{v} \in X_{R}} P\left(X_{v} \mid X_{p a(v)}\right) \sum_{w \in V_{L}} l\left(X_{p a(w)}\right)
$$

where $E L(X)$ is the expected loss across entire supply network

1) Standard configuration (SC): Supply network is considered to be in its standard configuration when all the control strategies selected in the BBN reflect real-time profile of these strategies in the supply network.

2) Contingency configuration (CC): Supply network is considered to be in its contingency configuration when all the control strategies in the BBN are selected against the multicriteria decision making of maximizing weighted summation of expected utility corresponding to loss exposure and utility of mitigation cost.

\section{B. Risk Measures}

1) Loss Propagation Containment Measure (LPCM): Loss propagation containment measure is the ratio between relative improvement in the network expected loss corresponding to complete mitigation of the risk factor and network expected loss for the standard configuration.

$$
\begin{aligned}
& L P C M_{X_{R_{i}}}=\frac{E L(X)-E L\left(X \mid X_{R_{i}}=\text { false }\right)}{E L(X)_{S C}} \\
& \text { Avg. } L P C M(\overline{L P C M})=1 / n \sum_{1}^{n} L P C M_{X_{R_{i}}}
\end{aligned}
$$

2) Loss Propagation Spread Measure (LPSM): Loss propagation spread measure is the ratio between range of network expected loss corresponding to the two extreme states of the risk factor and network expected loss for the standard configuration.

$$
\operatorname{LPSM}_{X_{R_{i}}}=\frac{E L\left(X \mid X_{R_{i}}=\text { true }\right)-E L\left(X \mid X_{R_{i}}=\text { false }\right)}{E L(X)_{S C}}
$$




$$
\operatorname{Avg} \cdot \operatorname{LPSM}(\overline{\operatorname{LPSM}})=1 / n \sum_{1}^{n} \operatorname{LPSM}_{X_{R_{i}}}
$$

\section{Problem Statement}

Given different levels of control strategies and associated costs available at different nodes of the supply network, what is the optimal combination of these control strategies yielding maximum utility for the decision maker?

\section{Objective Function}

The objective function is to select a specific combination of control strategies that would maximize the weighted sum of normalized expected utility for loss exposure and normalized utility of cost related to implementing control strategies for the decision maker.

$$
\begin{gathered}
\underset{\gamma_{x_{S}} \in \gamma_{X_{S}}}{\max _{X}} w * \overline{E U}\left(X_{\gamma_{x_{S}}}\right)+(1-w) * \bar{U}\left(C_{\gamma_{x_{S}}}\right) \\
E U(X)=\prod_{X_{v} \in X_{R}} P\left(X_{v} \mid X_{p a(v)}\right) \sum_{w \in V_{U}} u\left(X_{p a(w)}\right)
\end{gathered}
$$

where $\gamma_{X_{S}}$ is a set of all possible orderings of different states of $\mathrm{n}$ control strategies $\left(x_{s_{1}} \times x_{s_{2}} \times \ldots \times x_{s_{n}}\right)$

$w$ is the relative importance of the utility for loss exposure

$\overline{E U}(X)$ is the normalized expected utility of the decision maker for loss exposure

$C_{\gamma_{x_{S}}}$ is the cost of implementing $\gamma_{x_{S}}$ combination of control strategies
$\bar{U}(C)$ is the normalized utility of cost related to implementing control strategies

\section{An ILlustrative EXAMPLE}

We have used the same hypothetical supply network that was presented in a recent study [15] and modelled it using GeNIe [16] as shown in Fig. 1. The supply network comprises 6 elements and 12 associated risk factors. Supply network elements comprise combination of physical locations and transportation links. Risk factors representing uncertain events are shown as oval shaped nodes. Nomenclature appearing at the top of each risk factor represents its unique risk number followed by the respective supply network element (shown in parenthesis). Raw material source (RM) provides material to each of the two manufacturers (M1 and M2). The manufacturers supply finished goods to the warehouse (W) that are further transported to a retailer (R) through the transportation link of W-R. All risk factors except 'flood' (R12) are connected with discrete decision nodes (rectangle shaped) representing control strategies having two states of 'Yes' and 'No'. Loss value (represented by a diamond shaped node) of each risk event is same for the two states of control strategy; however, implementation of a control strategy reduces the (conditional) probability values of the relevant risk factor at some cost. (Conditional) probability and loss values for each risk factor are shown in Table I. Shaded cells represent (conditional) probability values corresponding to the 'Yes' state of associated control strategy. Three models were developed representing different risk attitudes of a decision maker assuming values shown in Table II.

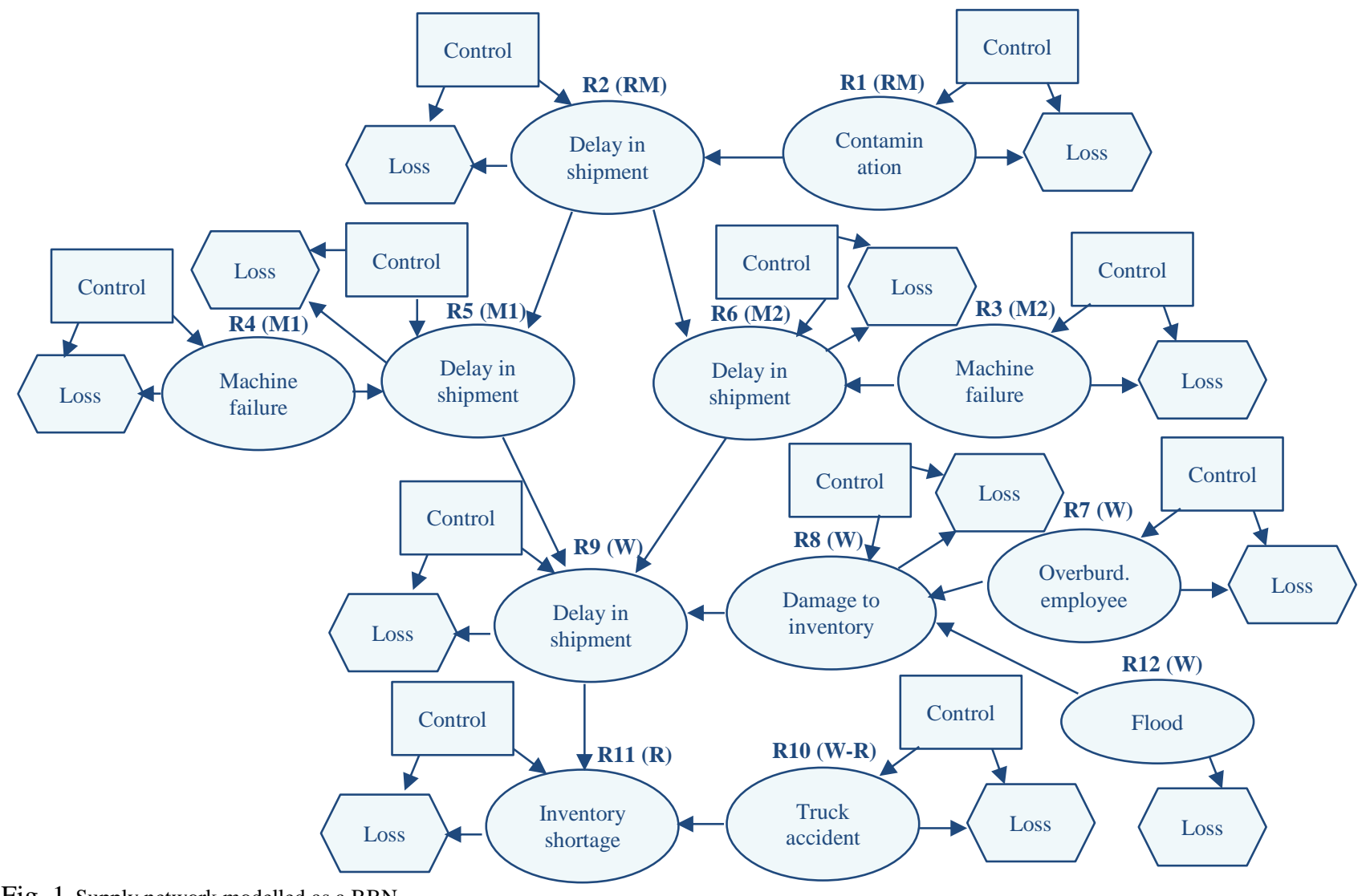

Fig. 1. Supply network modelled as a BBN. 
TABLE I. (CONDITIONAL) PROBABILITY AND LOSS VALUES

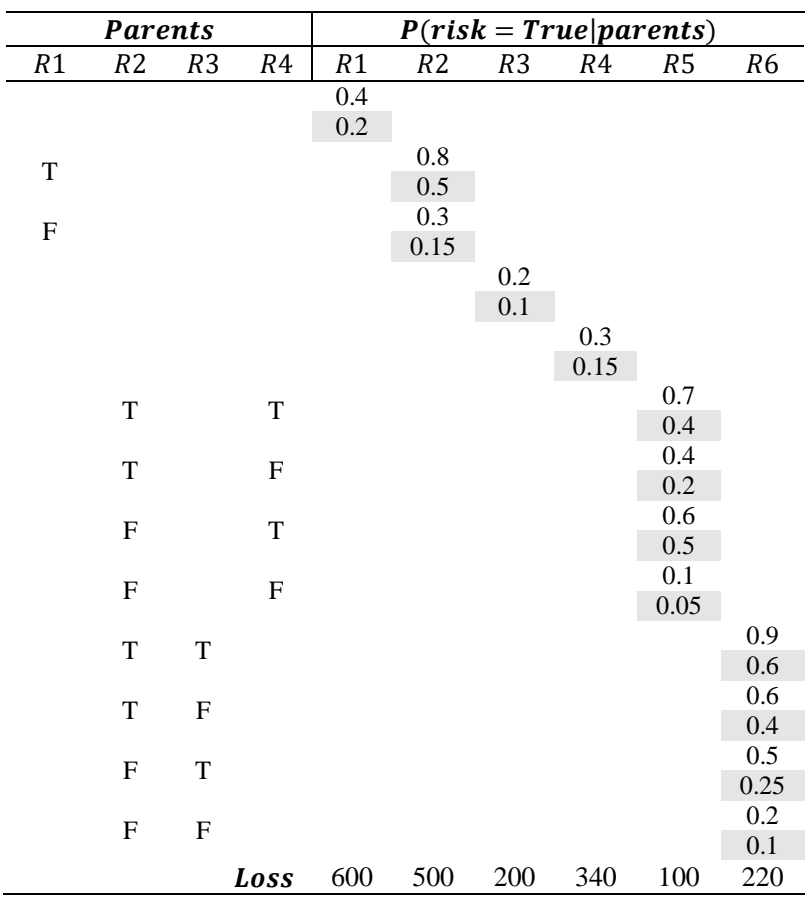

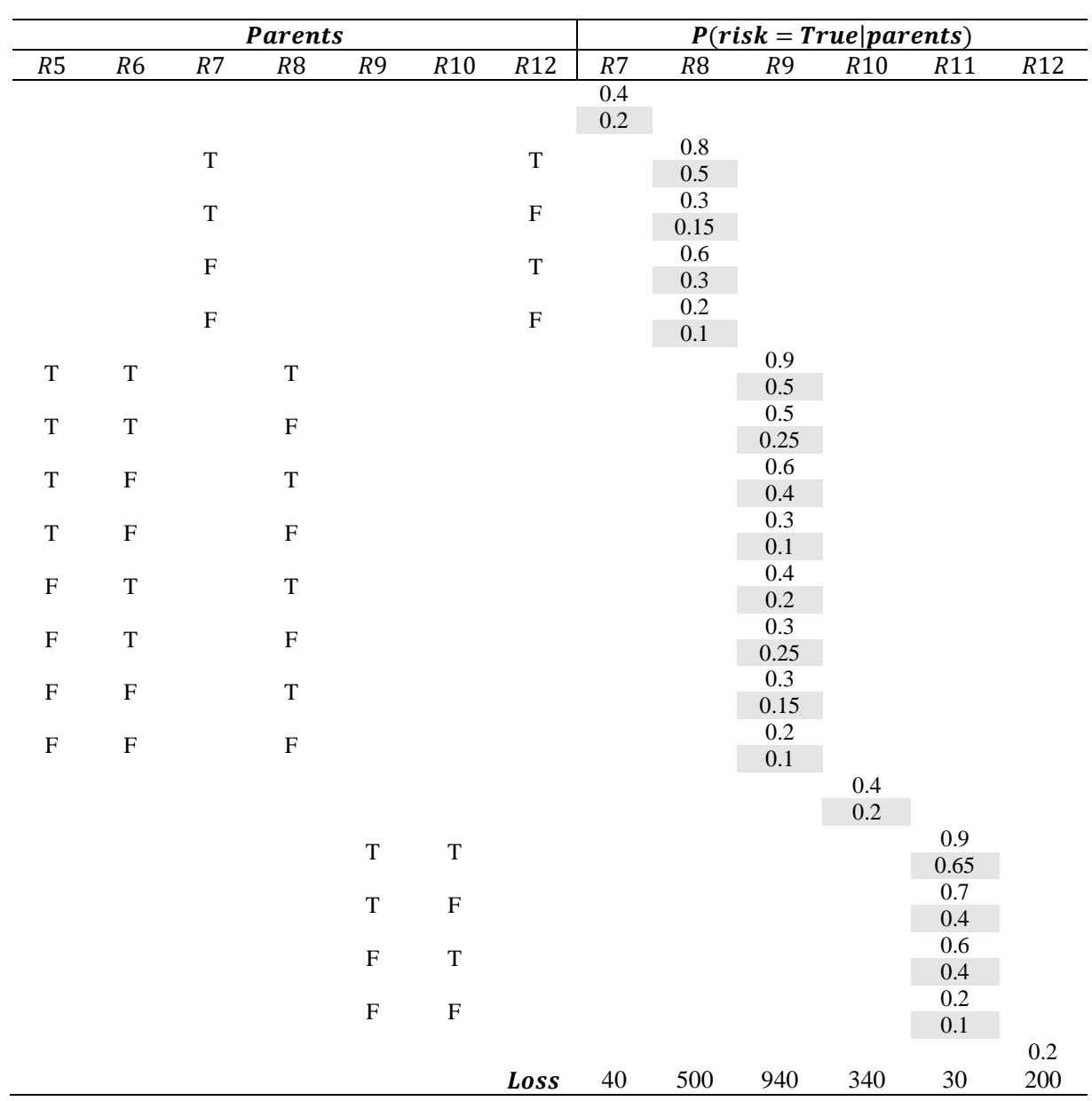


TABLE II. ASSUMED PARAMETERS

\begin{tabular}{cc}
\hline Parameter & Assumption \\
\hline$w$ & 0.5 \\
$u(x)$ for risk - neutral & $-x$ \\
$u(x)$ for risk - taking & $-x^{2}$ \\
$u(x)$ for risk - averse & $-\sqrt{x}$ \\
$\bar{U}(C)$ & $1-$ cost $/$ max cost \\
\hline
\end{tabular}

\section{RESULTS AND DISCUSSION}

After running the models corresponding to three different risk attitudes of the decision maker, values were generated in GeNIe and subsequently exported to Microsoft Excel for evaluation of risk measures and utility values concerning various combinations of control strategies. In case of a riskneutral decision maker, maximizing combination of expected utility for loss and utility for strategy cost resulted in the selection of control strategies as shown in Table III. Values of the risk measures for all risk factors under contingency configuration are graphically represented as Fig. 2. It is pertinent to mention that the total mitigation cost for the optimal combination can be allocated in different ways across the network and all such combinations except the optimal one would be inefficient as shown in Fig. 3.

A risk factor having low values for both the measures is considered as insignificant in terms of risk management as it will not have a major impact on the network in case of disruption and furthermore, the network will not benefit much from the its improvement as well. R7 can be categorized as the least important risk factor within the cohort. A risk factor having greater values against the two measures must be critically monitored and a control strategy implemented. R9 is the most important risk factor having maximum values against the risk measures. It is also counter-intuitive to note that $\mathrm{R} 9$ is not considered for implementing a control strategy although it is scored as the top ranking risk factor. This unique result manifests the importance of our modelling scheme which takes into consideration different factors including probabilistic interdependency between risks, resulting losses, impact of control strategies and associated cost.

TABLE III. OPTIMAL COMBINATION OF CONTROL STRATEGIES (RISKNEUTRAL DECISION MAKER)

\begin{tabular}{ccc}
\hline Control No & Cost & Implement \\
\hline 1 & 100 & Yes \\
2 & 150 & Yes \\
3 & 50 & No \\
4 & 100 & Yes \\
5 & 30 & Yes \\
6 & 80 & No \\
7 & 10 & Yes \\
8 & 150 & No \\
9 & 460 & No \\
10 & 80 & Yes \\
11 & 20 & No \\
\hline \multicolumn{2}{c}{ Total Mitigation Cost } & 470 \\
\hline Network Expected Loss & 970.07 \\
\hline
\end{tabular}

Average LPCM represents the average value of LPCM for all the risk factors. Variation of this important measure is shown in Fig. 4 with respect to all possible combinations of available control strategies. As there are 11 control strategies available across the supply network with each strategy having two states, there are a total of 2048 different combinations of control strategies. The graph represents average LPCM values for all these 2048 strategies. Corresponding to a specific budget allocation for implementing a control strategy, there is a unique optimal combination of resource allocation across the network in order to achieve the minimum value of average LPCM. All such points are shown in red colour representing the minimum points against specific mitigation cost. It is also evident from the graph that average LPCM value starts increasing close to 770 units of mitigation cost and gets back to the same value close to 1000 units of cost. Therefore, it will not be feasible to invest between the range of costs keeping into account the main objective of improving the average value of LPCM.

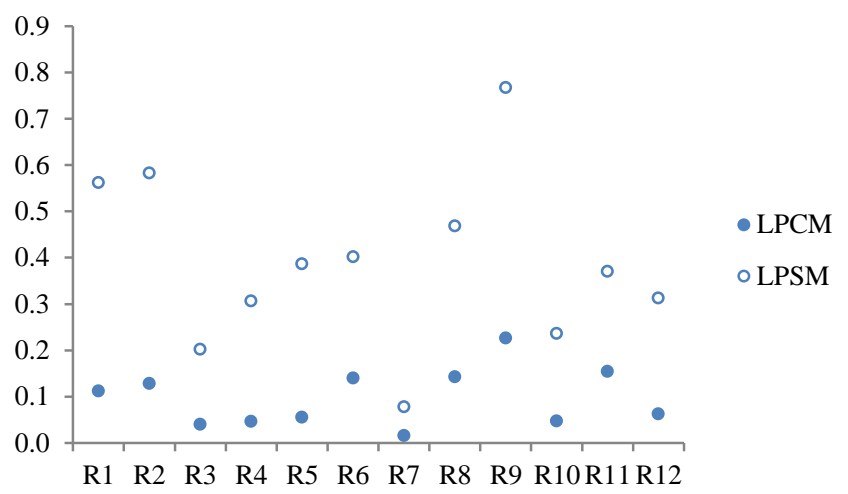

Fig. 2. Risk measures of the supply network under contingency configuration (risk-neutral decision maker).

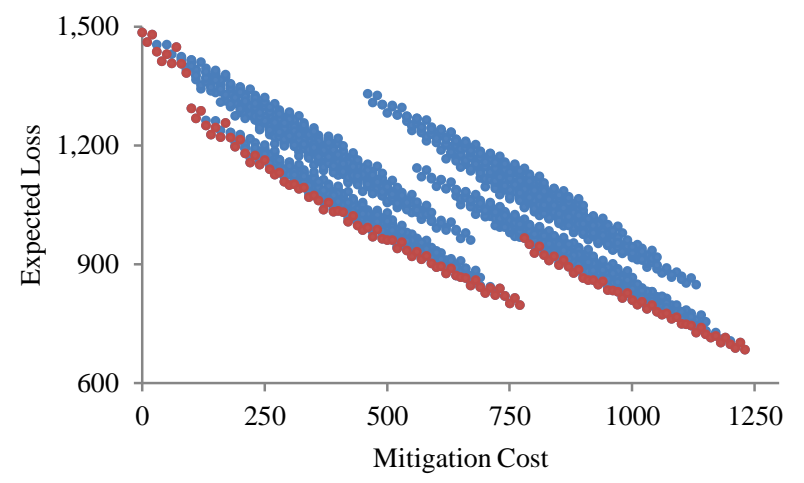

Fig. 3. Variation of network expected loss with all possible combinations of control strategies. 


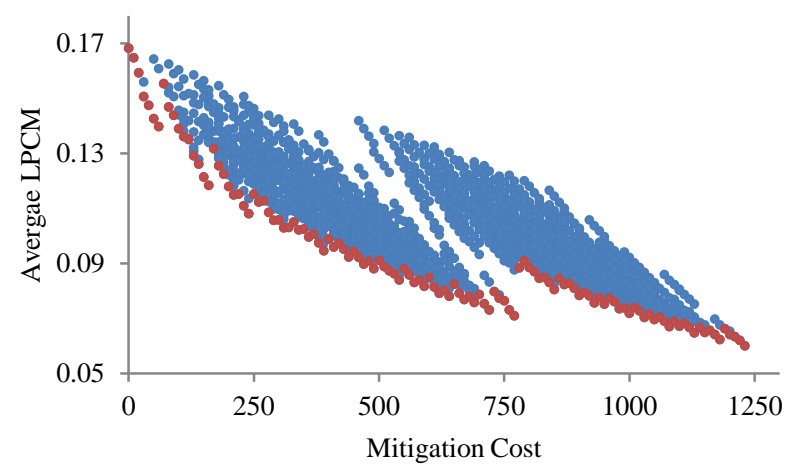

Fig. 4. Variation of average LPCM with all possible combinations of control strategies.

Average LPSM represents the average value of LPSM for all the risk factors. Variation of this important measure is shown in Fig. 5 with respect to all possible combinations of available control strategies. Corresponding to a specific budget allocation for implementing a control strategy, there is a unique optimal combination of resource allocation across the network in order to achieve the minimum value of average LPSM. All such points are shown in red colour representing the minimum points against specific mitigation cost. The rate of improvement of this measure declines with the increase in mitigation cost. Average LPSM represents the extent to which the network is protected against the potential disruptions and the decrease in this measure implies reduction in the dependency between risks across the network.

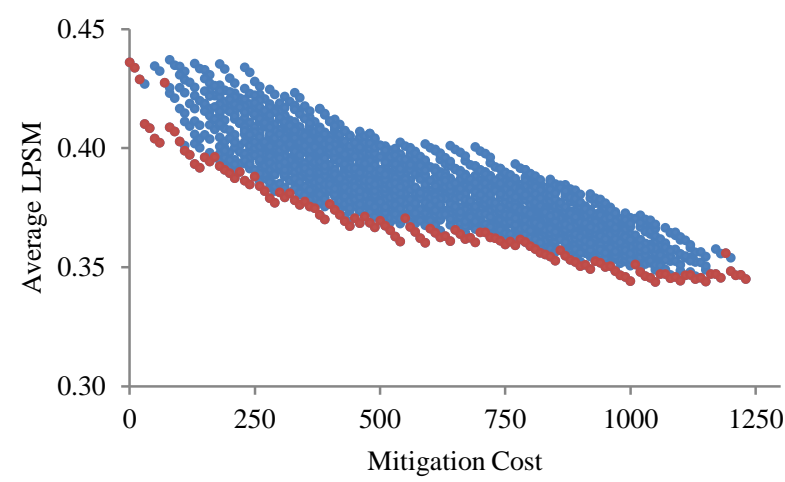

Fig. 5. Variation of average LPSM with all possible combinations of control strategies.

Utility of the risk-neutral decision maker was calculated against all possible combinations of control strategies as shown in Fig. 6. It is evident from the graph that there can be a number of combinations of control strategies against specific budget allocation; however, only a unique combination results in maximizing the utility value. Red coloured points represent all such combinations that maximize the utility values against specific mitigation cost. The maximum utility value is 0.63 corresponding to the total mitigation cost of 470 units.

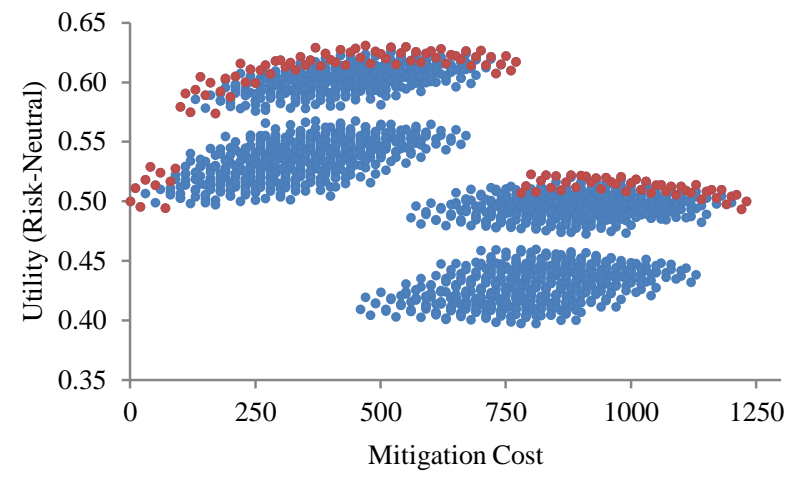

Fig. 6. Variation of utility for risk-neutral decision maker with all possible combinations of control strategies.

Utility of the risk-averse decision maker was calculated against all possible combinations of control strategies as shown in Fig. 7. Red coloured points represent all combinations of control strategies that maximize the utility values against specific mitigation cost. The maximum utility value is 0.65 corresponding to the mitigation cost of 540 units. It is important to consider that equal weightage was assigned to the two attributes of utility of cost and expected utility of loss. Risk-averse decision makers might allocate higher weightage to the expected utility of loss and it might influence the results.

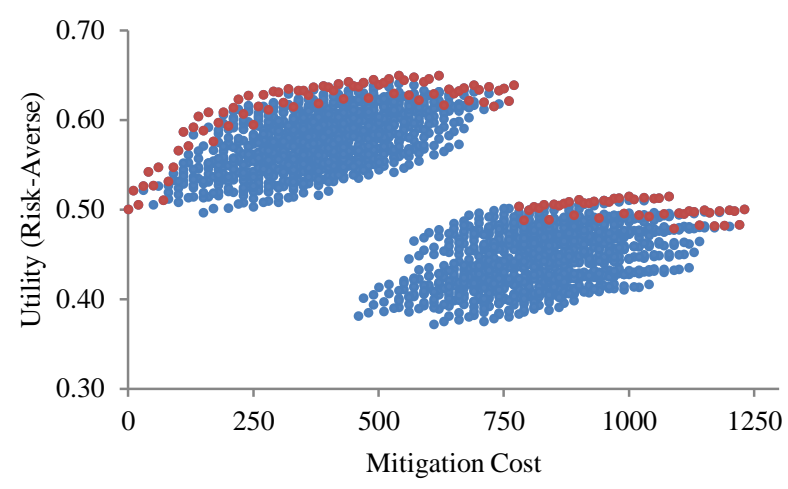

Fig. 7. Variation of utility for risk-averse decision maker with all possible combinations of control strategies.

Utility of the risk-taking decision maker was calculated against all possible combinations of control strategies as shown in Fig. 8. Red coloured points represent all combinations of control strategies that maximize the utility values against specific mitigation cost. The maximum utility value is 0.62 corresponding to the mitigation cost of 440 units. It is important to consider that equal weightage was assigned to the two attributes of utility of cost and expected utility of loss. Risk-taking decision makers might allocate lower weightage to the expected utility of loss and it might influence the results. In contrast to the previous two graphs, there is a decreasing trend in the maximum utility values with increase in mitigation cost 
and it can be justified on the basis of low importance of expected loss for the risk-taking decision maker.

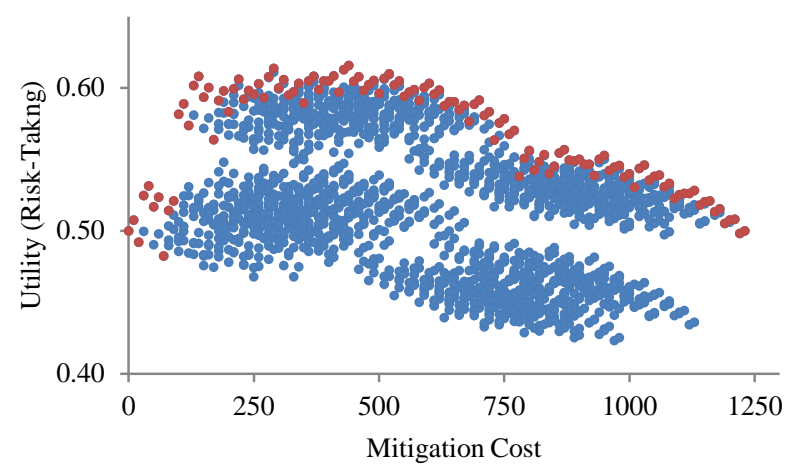

Fig. 8. Variation of utility for risk-taking decision maker with all possible combinations of control strategies.

Maximum utility values for all three risk attitudes of the decision maker are plotted against mitigation cost in Fig. 9. There is a discontinuity close to 770 units of mitigation cost because certain mitigation control implemented at that cost might not be included in the combinations considered beyond that cost and once it is included again in the package, the benefits would realize but the cost would outweigh these benefits. For risk-taking decision maker, the utility starts declining earlier. Furthermore, for the first part of graph before the discontinuity, risk-averse decision maker generally gets higher utility values.

It is also important to consider the variation of maximum expected utility values for loss and utility for cost corresponding to three different risk attitudes with mitigation cost as shown in Fig. 10. Utility function of cost was assumed as linear. For risk-neutral decision maker, expected utility graph for loss is the mirror reflection of expected loss graph. For costs below 770 units, risk-taking decision maker generally gets the least utility values; however, after the discontinuity, the situation is different because there is a sharp increase in expected loss that increases the disutility for the other types of decision maker more than that for the risk-taking one.

We have proposed new risk measures and a novel method of managing supply chain risks taking into consideration all three stages of risk management. It is important to consider the loss values and mitigation strategies including associated costs within the model. Our proposed method also captures risk attitude of the decision maker as the stakeholders might have different attitudes towards managing risks. The method can be used for gauging the importance of risk factors and supply network elements under standard configuration and selecting optimal combination of control strategies under contingency configuration. We have bridged the research gap of using BBNs in SCRM encompassing all three stages of risk management; risk identification, risk assessment and risk evaluation.

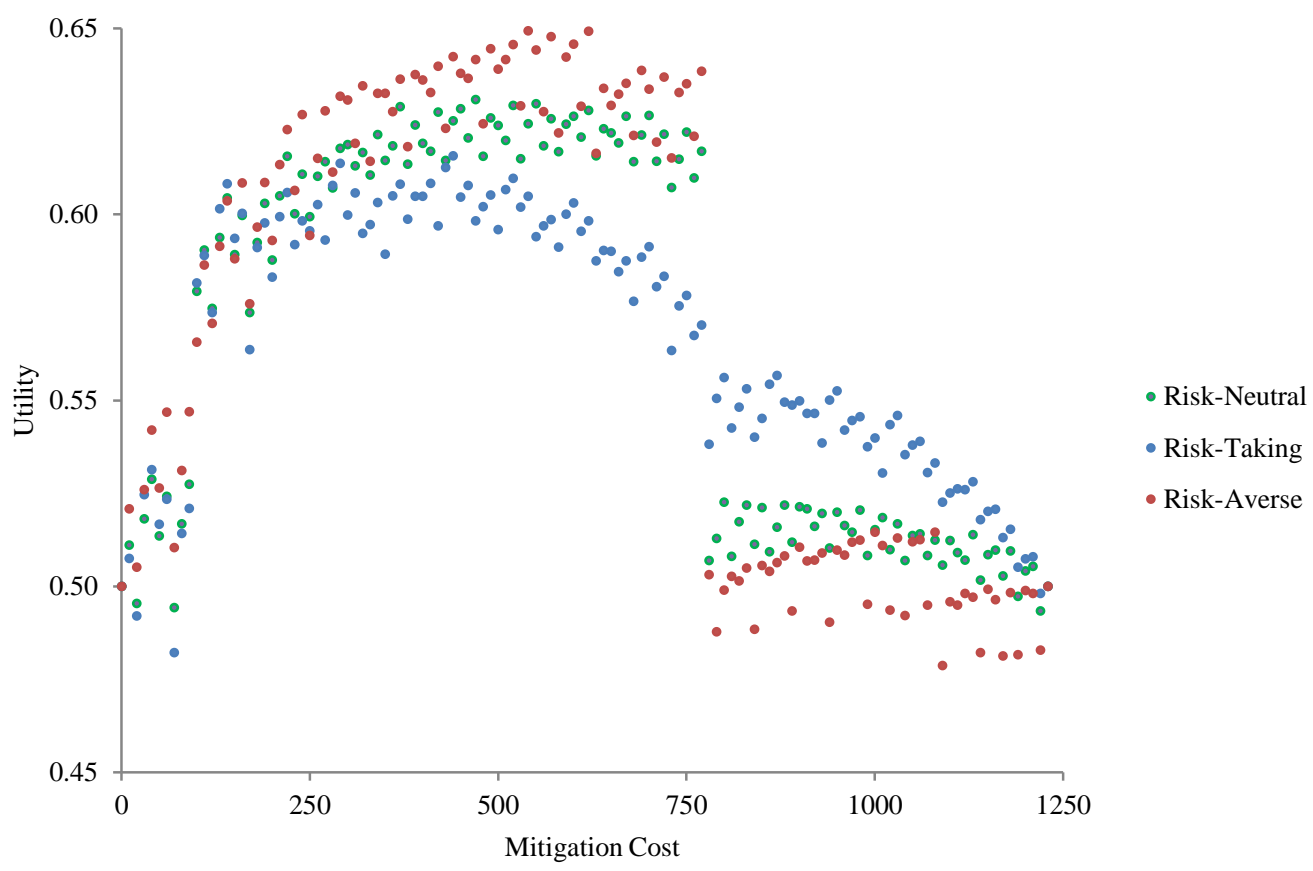

Fig. 9. Variation of maximum utility values for three different risk attitudes with mitigation cost. 


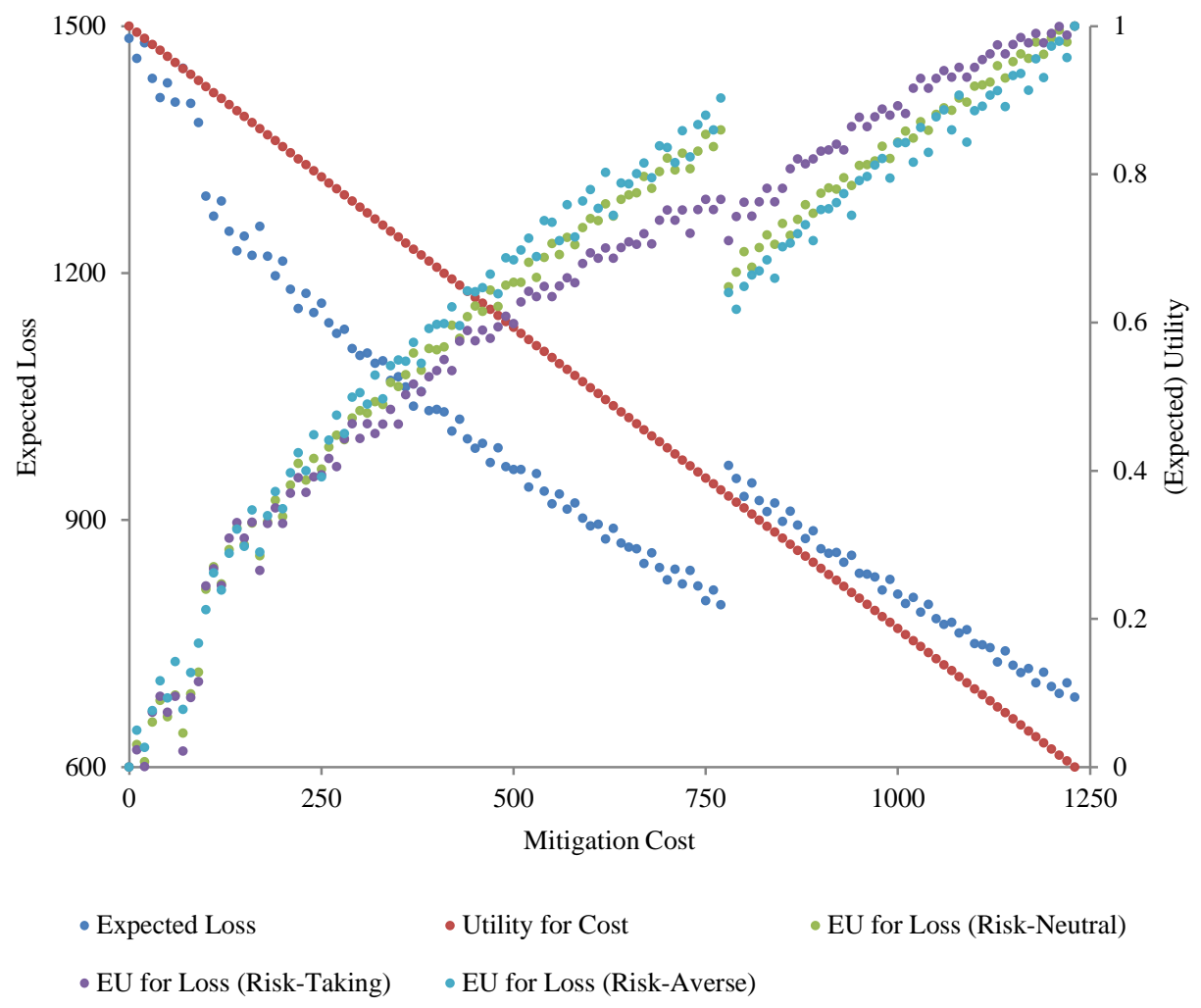

Fig. 10. Variation of maximum expected utility values for loss and utility for cost corresponding to three different risk attitudes with mitigation cost.

\section{CONCLUSION}

Supply chain risk management is an active area of research. Researchers are developing robust and effective techniques in order to manage the complex interaction of risk factors across the global supply chains. Bayesian networks have been applied in specific domains of supply chain risk management; however, the existing models do not encompass all three stages of risk management. We have proposed modelling a supply network as a Bayesian belief network capturing network configuration, probabilistic interdependency between risk factors, resulting losses, control strategies and associated costs. Our proposed risk measures represent the importance of risk factors in terms of their contribution towards the loss propagation across the entire network.

We were able to demonstrate the application of our proposed method through modelling a simple illustrative supply network. The modelling approach is considered to be an important contribution to the existing literature as it bridges the research gap of modelling interdependency between supply chain risks encompassing all stages of risk management. Our model can also facilitate solving different objective functions in accordance with the risk attitude of decision maker. It is evident from our results that it is only possible to evaluate the merits of different combinations of risk mitigation strategies after considering the respective costs into the model and without adopting such approach and relying only on the probabilistic interaction might not help in taking optimal decisions. Future research might focus on the application of this modelling approach in a real case study.

\section{REFERENCES}

[1] Handfield, R., Blackhurst, J., Craighead, C.W., and Elkins, D. Introduction: a managerial framework for reducing the impact of disruptions to the supply chain. 2011.

[2] Manuj, I. and Mentzer, J.T., Global supply chain risk management strategies. International Journal of Physical Distribution and Logistics Management, 2008. 38(3): pp. 192-223.

[3] Badurdeen, F., Shuaib, M., Wijekoon, K., Brown, A., et al., Quantitative modeling and analysis of supply chain risks using Bayesian theory. Journal of Manufacturing Technology Management, 2014. 25(5): pp. 631-654.

[4] Sigurdsson, J.H., Walls, L.A., and Quigley, J.L., Bayesian belief nets for managing expert judgement and modelling reliability. Quality and Reliability Engineering International, 2001. 17(3): pp. 181-190.

[5] Kelangath, S., Das, P.K., Quigley, J., and Hirdaris, S.E., Risk analysis of damaged ships - a data-driven Bayesian approach. Ships and Offshore Structures, 2011. 7(3): pp. 333-347.

[6] Qazi, A., Quigley, J., and Dickson, A. A novel framework for quantification of supply chain risks. in 4th Student Conference on Operational Research. 2014. University of Nottingham, UK.

[7] Tang, C.S., Perspectives in supply chain risk management. International Journal of Production Economics, 2006. 103(2): pp. 451-488.

[8] Jüttner, U., Peck, H., and Christopher, M., Supply chain risk management: outlining an agenda for future research. International Journal of Logistics Research and Applications, 2003. 6(4): pp. 197-210.

[9] Christopher, M. and Peck, H., Building the Resilient Supply Chain. International Journal of Logistics Management, 2004. 15(2): pp. 1-14.

[10] Qazi, A., Quigley, J., and Dickson, A., Supply Chain Risk Management: Systematic literature review and a conceptual framework for capturing 
interdependencies between risks, in 5th International Conference on Industrial Engineering and Operations Management. 2015: Dubai.

[11] Lockamy, A. and McCormack, K., Modeling supplier risks using Bayesian networks. Industrial Management \& Data Systems, 2012. 112(2): pp. 313-333.

[12] Lockamy, A., Benchmarking supplier risks using Bayesian networks. Benchmarking: An International Journal, 2011. 18(3): pp. 409-427.

[13] Lee, E., Park, Y., and Shin, J.G., Large engineering project risk management using a Bayesian belief network. Expert Systems with Applications, 2009. 36(3, Part 2): pp. 5880-5887.
[14] Dogan, I. and Aydin, N., Combining Bayesian Networks and Total Cost of Ownership method for supplier selection analysis. Computers and Industrial Engineering, 2011. 61(4): pp. 1072-1085.

[15] Garvey, M.D., Carnovale, S., and Yeniyurt, S., An analytical framework for supply network risk propagation: A Bayesian network approach. European Journal of Operational Research, 2015. 243(2): pp. 618-627.

[16] The Decision Systems Laboratory, GeNIe and SMILE. [accessed on 5 March, 2015]; Available from: http://genie.sis.pitt.edu/about.html. 